\title{
Poor prognosis in young patients with atrioventricular block of unknown aetiology: who is to blame? The physician or the pace-maker?
}

\section{Editorial}

Editorial

\section{(1) Raphaël P. Martins ${ }^{1}$, Alban-Elouen Baruteau ${ }^{2}{ }^{3}$ and J-Claude Daubert ${ }^{1 *}$ claude.dau- bert@univ-rennes1.fr}

1. Université de Rennes, CHU Rennes, INSERM, LTSI-UMR 1099, Rennes, France

2. Institut du thorax, INSERM, CNRS, Université de Nantes, Nantes, France

3. Department of Pediatric Cardiology, Children's Hospital, CHU Nantes, France

The opinions expressed in this article are not necessarily those of the Editors of the European Heart Journal or of the European Society of Cardiology.

${ }^{*}$ Corresponding author. Faculté de Médecine, Université de Rennes 1, 35043, Rennes, France. Email: claude.daubert@univ-rennes1.fr

This editorial refers to 'Long-term outcomes in young patients with atrioventricular block of unknown aetiology', by J. Resdal Dideriksen et al., on page xxx.

The rate of permanent pacemaker (PPM) implantation is still growing in Europe, due in part to population ageing with an increased risk of developing conduction diseases. Although most PPM recipients are elderly patients, some young patients experience atrioventricular block (AVB) requiring PPM. In a recent study by Rudbeck-Resdal et al. evaluating AVB in young patients, the estimated incidence of developing AVB before 50 years of age was 17.7/year/ million inhabitants throughout their 20 -year nationwide study period. ${ }^{1}$ The aetiology of AVB was identified in only half of the patients. The most frequent causes were complications to cardiac surgery $(15.3 \%)$, congenital AVB or congenital heart disease $(9.0 \%$ and $4.2 \%$, respectively), and cardioinhibitory reflex $(5.0 \%)$. An underlying cardiomyopathy or sarcoidosis were uncommon causes in this specific population.

Graphical Abstract Atrioventricular block of unknown origin in the young: who is responsible? CMR, cardiac magnetic resonance. 


\section{Atrioventricular block of unknown origin in the young}

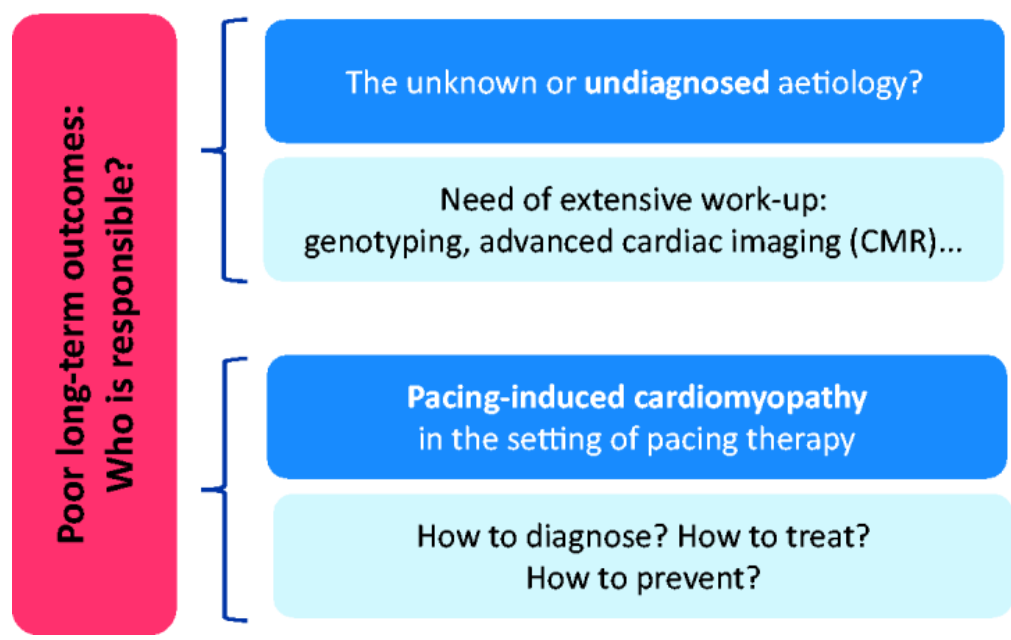

In this issue of the European Heart Journal, Resdal Dideriksen et al. present a new analysis focusing on long-term clinical outcomes in their population of young patients ( $<50$ years) with PPM implanted for AVB of unknown aetiology. $^{2}$ The authors report original data from a retrospective, case-control observational study, over a median follow-up of 9.8 years, and show a 3.8-fold higher rate of death, or hospitalization for heart failure (HF), ventricular tachyarrhythmias, or aborted sudden death, compared with an age- and sex-matched control population. A higher risk was found in patients with permanent AVB at the time of implantation, and during the first 5 years after implantation. These interesting but somewhat disturbing data raise a number of questions about what exactly is meant by 'unknown aetiology' and the potential role of pacing therapy in the occurrence of adverse outcomes.

\section{Unknown aetiologies and relationship to clinical outcomes}

In the study by Resdal Dideriksen et al., the 50.3\% rate of AVB from unknown aetiology emphasizes the lack of thorough case investigations in this cohort. ${ }^{2}$ The poor outcome observed in these patients may actually be due to an underlying disease, which is unknown because it has been insufficiently investigated, i.e. appropriate data on genotyping and advanced cardiac imaging are lacking. The low $0.6 \%$ rate of genetic $\mathrm{AVB}^{2}$ is due to limited genetic testing in the studied population, as SCN5A mutations account for $\sim 5 \%$ of cardiac conduction disorders in the young. ${ }^{3} \mathrm{Ge}-$ netic testing should be considered in patients with early-onset (age $<50$ years) cardiac conduction disorders, either isolated or associated with structural heart disease, ${ }^{3}$ as genetic variants in multiple genes have been described. Here, genetic mutations with a strong impact on patients' outcomes, such as LMNA mutations or SCN5A mutations possibly in the setting of cardiac sodium channelopathy overlap syndrome, ${ }^{4}$ may have been missed.

Autoantibody-mediated AVB may have been underestimated as well. The authors did not systematically check the immune status of the patients' mothers using high sensitivity, quantitative radioligand assays, although it has been reported that $\sim 10 \%$ of mothers who are sero-negative for anti-Ro/SSA and anti-La/SSB at the time of foetal diagnosis later become sero-positive. This is a striking point as well, as immune-mediated AVB patients are exposed to dilated cardiomyopathy and death, ${ }^{5}$ which contrasts with the favourable long-term prognosis of 'true' idiopathic AVB diagnosed in the young. 6

Lastly, cardiac magnetic resonance imaging was not performed on a systematic basis to rule out ischaemic heart disease, myocarditis, sarcoidosis, or amyloidosis, although this imaging modality has proved to have an incremental diagnostic role when added to standard clinical assessment. ${ }^{7}$

\section{Role of pacing therapy}

As stated by the authors, the poor outcomes observed may also be the consequence of pacing therapy, through socalled 'pacing-induced cardiomyopathy' (PICM). This concept, first introduced by Karpawich et al. ${ }^{8}$ in the late 
1990s, has generated an abundant literature summarized in a recent review article. ${ }^{9}$ In the present study, ${ }^{2}$ data on left ventricular ejection fraction (LVEF) and pacing burden during follow-up are not available, and the study cannot conclude whether the poor outcomes observed were driven by the development of PICM. In the general PPM population, the incidence of PICM varies between $10 \%$ and $20 \%$ among published series, depending on the definition used (echocardiographic, with an absolute LVEF decrease $>10 \%$ or impairment of LVEF below 50\%; or clinical, as new-onset $\mathrm{HF})$ and the duration of follow-up. Recent data from a large pacemaker registry suggest that the risk of new-onset HF is higher in patients implanted for AVB, with the highest incidence in the younger age group ( $<55$ years). ${ }^{10}$ The real mechanisms underlying PICM are not fully understood, but include heterogeneous electrical activation, mimicking left bundle branch block, mechanical activation changes, modifications of regional myocardial perfusion, and histological modifications. These structural and functional changes explain the impaired regional contraction in paced patients, near the pacing site. Overall systolic and diastolic functions can significantly decrease, with abnormalities already visible after 18 months of follow-up. Adverse left ventricular (LV) remodelling may occur, leading to overt HF.

Therefore, how can we avoid such a poor prognosis in young PPM recipients, as observed in this study?

Firstly, a detailed diagnostic work-up should be undertaken to recognize the maximum potential high-risk aetiologies, including the patient's personal medical history, family history of cardiomyopathy, echocardiography, laboratory testing to rule out borreliosis, molecular genetic testing to rule out muscular dystrophy and hereditary causes, and cardiac magnetic resonance imaging. ${ }^{1}$ This is especially important because some particular aetiologies have a significant impact on patient management and outcome. For instance, current guidelines recommend the implantation of an implantable cardioverter-defibrillator in cardiac sarcoidosis patients presenting with AVB since they are at high risk of ventricular arrhythmias and sudden cardiac death. ${ }^{11}$

Secondly, one should determine whether a given patient is at risk of developing PICM and consequently try to prevent it. The most obvious predictor of PCIM is baseline LVEF before implantation. Indeed, if LV function is already impaired, even in asymptomatic patients, adding a non-physiological ventricular activation may further compromise LVEF and lead to overt HF. The BLOCK-HF trial randomized patients with complete AVB, LVEF $\leq 50 \%$, and New York Heart Association (NYHA) class I-III to standard right ventricular (RV) pacing or biventricular (BiV) pacing. ${ }^{12}$ The primary outcome was time to death from any cause, an urgent care visit for $\mathrm{HF}$, or a $>15 \%$ increase in LV end-systolic volume index. A primary outcome event occurred in $55.6 \%$ and $45.8 \%$ of patients in the RV and BiV pacing groups, respectively (hazard ratio $0.74,95 \%$ confidence interval $0.60-0.90$ ). Hospitalization and mortality rates were $30 \%$ and $17 \%$ lower, respectively, in the BiV pacing group. The authors concluded that BiV pacing is superior to conventional RV pacing in patients with AV block, LV dysfunction, and NYHA class I-III symptoms.

Other factors such as age, male gender, renal impairment, diuretic use at baseline, and pre-implantation QRS width were found to be predictors of PICM.

An optimal pacing site that produces as much physiological ventricular activation as possible should also be chosen to avoid LV dysfunction. Indeed, ventricular pacing, particularly if performed at the RV apex, will cause an asynchronous LV activation, potentially leading to HF. Of note, $61 \%$ of the patients included in the study of Resdal Dideriksen et al. ${ }^{2}$ had apical leads, possibly contributing to the impaired outcomes observed. Furthermore, many studies evaluating the long-term effects of RV apical pacing supported the concept that the higher the pacing burden (\%), the more likely it is that LV dysfunction and HF will occur. The MOST trial enrolled patients with sinus node dysfunction needing PPM, and showed that the risk of HF hospitalization increased by $20 \%$ for every $10 \%$ increase in RV pacing. Pacing burden $>40 \%$ was associated with a 2.6 -fold increased risk of HF hospitalization in patients with DDDR devices, compared with those with $<40 \%$ of RV pacing. ${ }^{13}$

Consequently, alternative pacing sites have been tested, e.g. RV septum, RV outflow tract, LV only pacing, etc. His bundle pacing could be a preferential option, ${ }^{14}$ but it still needs to be assessed in large randomized controlled studies.

In patients with PICM, one option before scheduling BiV or His bundle pacing upgrade could be to assess RV pacing burden and evaluate whether a simple reprogramming could be sufficient to decrease the pacing burden and reverse LV dysfunction. ${ }^{15}$ 
The study by Resdal Dideriksen et al. ${ }^{2}$ adds new and important findings for the community of device physicians, even though they are hypothesis generating. Whether the poor outcomes observed are due to the implanting physician (because of a lack of thorough work-up to find a possible aetiology for the AVB associated with poor outcomes) or to the device itself (through a PICM mechanism) is still a matter of debate and would require further studies (Graphical Abstract).

The authors should be commended for their work emphasizing the necessity of a detailed work-up before device implantation in patients aged $<50$ years and improved follow-up strategies to prevent the occurrence of adverse outcomes.

Conflict of interest: none declared.

\section{References}

1. Rudbeck-Resdal J, Christiansen MK, Johansen JB, Nielsen JC, Bundgaard H, Jensen HK. Aetiologies and temporal trends of atrioventricular block in young patients: a 20-year nationwide study. Europace 2019;21:1710-1716.

2. Resdal Dideriksen J, Christiansen MK, Johansen JB, Nielsen JC, Bundgaard H, Jensen HK. Long-term outcomes in young patients with atrioventricular block of unknown aetiology. Eur Heart J 2021;42:ehab060.

3. Priori SG, Wilde AA, Horie M, Cho Y, Behr ER, Berul C, Blom N, Brugada J, Chiang CE, Huikuri H, Kannankeril P, Krahn A, Leenhardt A, Moss A, Schwartz PJ, Shimizu W, Tomaselli G, Tracy C. HRS/EHRA/APHRS expert consensus statement on the diagnosis and management of patients with inherited primary arrhythmia syndromes: document endorsed by HRS, EHRA, and APHRS in May 2013 and by ACCF, AHA, PACES, and AEPC in June 2013. Heart Rhythm 2013;10:1932-1963.

4. Baruteau AE, Kyndt F, Behr ER, Vink AS, Lachaud M, Joong A, Schott JJ, Horie M, Denjoy I, Crotti L, Shimizu W, Bos JM, Stephenson EA, Wong L, Abrams DJ, Davis AM, Winbo A, Dubin AM, Sanatani S, Liberman L, Kaski JP, Rudic B, Kwok SY, Rieubland C, Tfelt-Hansen J, Van Hare GF, Guyomarc'h-Delasalle B, Blom NA, Wijeyeratne YD, Gourraud JB, Le Marec H, Ozawa J, Fressart V, Lupoglazoff JM, Dagradi F, Spazzolini C, Aiba T, Tester DJ, Zahavich LA, Beausejour-Ladouceur V, Jadhav M, Skinner JR, Franciosi S, Krahn AD, Abdelsayed M, Ruben PC, Yung TC, Ackerman MJ, Wilde AA, Schwartz PJ, Probst V. SCN5A mutations in 442 neonates and children: genotype-phenotype correlation and identification of higher-risk subgroups. Eur Heart J 2018;39:2879-2887.

5. Baruteau AE, Pass RH, Thambo JB, Behaghel A, Le Pennec S, Perdreau E, Combes N, Liberman L, McLeod CJ. Congenital and childhood atrioventricular blocks: pathophysiology and contemporary management. Eur J Pediatr 2016;175:1235-1248.

6. Baruteau AE, Fouchard S, Behaghel A, Mabo P, Villain E, Thambo JB, Marcon F, Gournay V, Rouault F, Chantepie A, Guillaumont S, Godart F, Bonnet C, Fraisse A, Schleich JM, Lusson JR, Dulac Y, Leclercq C, Daubert JC, Schott JJ, Le Marec H, Probst V. Characteristics and long-term outcome of non-immune isolated atrioventricular block diagnosed in utero or early childhood: a multicentre study. Eur Heart J 2012;33:622-629.

7. Baritussio A, Ghosh Dastidar A, Frontera A, Ahmed N, De Garate E, Harries I, Diab I, Duncan E, Thomas G, Nisbet A, Bucciarelli-Ducci C. Diagnostic yield of cardiovascular magnetic resonance in young-middle aged patients with high-grade atrio-ventricular block. Int J Cardiol 2017;244:335-339.

8. Karpawich PP, Rabah R, Haas JE. Altered cardiac histology following apical right ventricular pacing in patients with congenital atrioventricular block. Pacing Clin Electrophysiol 1999;22:1372-1377.

9. Merchant FM, Mittal S. Pacing induced cardiomyopathy. J Cardiovasc Electrophysiol 2020;31:286-292.

10. Merchant FM, Hoskins MH, Musat DL, Prillinger JB, Roberts GJ, Nabutovsky Y, Mittal S. Incidence and time course for developing heart failure with high-burden right ventricular pacing. Circ Cardiovasc Qual Outcomes 2017;10:e003564.

11. Birnie DH, Sauer WH, Bogun F, Cooper JM, Culver DA, Duvernoy CS, Judson MA, Kron J, Mehta D, Cosedis Nielsen J, Patel AR, Ohe T, Raatikainen P, Soejima K. HRS expert consensus statement on the diagnosis and management of arrhythmias associated with cardiac sarcoidosis. Heart Rhythm 2014;11:1305-1323. 
12. Curtis AB, Worley SJ, Adamson PB, Chung ES, Niazi I, Sherfesee L, Shinn T, Sutton MS; Biventricular versus Right Ventricular Pacing in Heart Failure Patients with Atrioventricular Block (BLOCK HF) Trial Investigators. Biventricular pacing for atrioventricular block and systolic dysfunction. N Engl J Med 2013;368:1585-1593.

13. Sweeney MO, Hellkamp AS, Ellenbogen KA, Greenspon AJ, Freedman RA, Lee KL, Lamas GA; MOde Selection Trial Investigators. Adverse effect of ventricular pacing on heart failure and atrial fibrillation among patients with normal baseline QRS duration in a clinical trial of pacemaker therapy for sinus node dysfunction. Circulation 2003;107:2932-2937.

14. Vijayaraman P, Herweg B, Dandamudi G, Mittal S, Bhatt AG, Marcantoni L, Naperkowski A, Sharma PS, Zanon F. Outcomes of His-bundle pacing upgrade after long-term right ventricular pacing and/or pacing-induced cardiomyopathy: insights into disease progression. Heart Rhythm 2019;16:1554-1561.

15. Gierula J, Jamil HA, Byrom R, Joy ER, Cubbon RM, Kearney MT, Witte KK. Pacing-associated left ventricular dysfunction? Think reprogramming first! Heart 2014;100:765-769. 